\title{
CHARACTERISTIC CLASSES OF HOMOGENEOUS SPACES
}

BY

\section{ALFRED ADLER}

Introduction. It is the object of this paper to prove the results of $\S \S 11$, 12 , and 13 ; for a homogeneous space $G / K$ ( $G$ a compact Lie group, $K$ a closed connected subgroup) it is shown that:

if $K$ is abelian, the Pontrjagin classes of $G / K$ are zero;

if $G / K$ is symmetric, or simply-connected complex homogeneous, all characteristic classes of degree greater than (dimension $G / K)-(\operatorname{rank} G-\operatorname{rank} K)$ are zero.

Finally, it is shown that the characteristic classes of $G / K$ depend, in a sense, only on the abelian part of $K$.

The canonical connection of the second kind on $G / K$ is used to demonstrate that the characteristic classes of $G / K$ with respect to its bundle of oriented frames can be considered characteristic classes of $G / K$ with respect to its principal $K$-bundle. The characteristic classes of the $K$-bundle structure are then computed by means of the well-known algebraic operations in the Lie algebra of a semi-simple Lie group.

The first sections of this paper are devoted to an exposition of the needed facts concerning characterisic classes, in particular those of homogeneous spaces; much of the material is based on [3]. The numbers in brackets refer to the bibliography.

I am much indebted to I. M. Singer for the frequent advice he has given me during the past year.

1. Invariants. Let $g$ be the Lie algebra of a Lie group $G$ of dimension $N$. A real-valued $r$-linear function $T$ on $\mathfrak{g}$ is called a symmetric $r$-tensor if $T(\cdots, X, \cdots, Y, \cdots)=T(\cdots, Y, \cdots, X, \cdots)$ for any two elements $X, Y$ of g. It is called invariant under $G$ (or, simply, invariant) if $T(\operatorname{ad} g X, \cdots, \operatorname{ad} g Z)=T(X, \cdots, Z)$ for any $r$ elements $X, \cdots, Z$ of $g$ and any element $g$ of $G$. The set of all invariant symmetric $r$-tensors on $g$ is denoted by $I^{r}(\mathfrak{g})$, and the set of all invariant symmetric tensors on $\mathfrak{g}$ is denoted by $I(\mathfrak{g})$.

If $T, T^{\prime}$ are symmetric $r$-tensors on $\mathfrak{g}$ and $S$ is a symmetric $s$-tensor on $\mathfrak{g}$, we define symmetric $r$ - and $r+s$-tensors $T+T^{\prime}$ and $T \cdot S$ on $g$ as follows:

$$
\begin{aligned}
&\left(T+T^{\prime}\right)(X, \cdots, Z)=T(X, \cdots, Z)+T^{\prime}(X, \cdots, Z), \\
&(T \cdot S)\left(X_{1}, \cdots, X_{r+s}\right)=((r+s) !)^{-1} \sum T\left(X_{i_{1}}, \cdots, X_{i_{r}}\right) S\left(X_{i_{r+1}}, \cdots, X_{i_{r+s}}\right),
\end{aligned}
$$

$X_{1}, \cdots, X_{r+\delta}$ being any elements of $g$ and the summation extending over all permutations $i_{1}, \cdots, i_{r+s}$ of $1, \cdots, r+s$. 
These operations define a commutative ring structure in the set of all symmetric tensors on $\mathfrak{g}$, and in fact this ring is isomorphic to the ring of all polynomials in $N$ variables. If $T, T^{\prime}$, and $S$ are invariant, so are $T+T^{\prime}$ and $T \cdot S$; hence the above operations define a ring structure in $I(\mathfrak{g})$.

Propositron. If $G$ is connected, then an r-linear real-valued function $f$ on $\mathrm{g}$ is invariant under $G$ if and only if the following equality holds for any choice of elements $X_{1}, \cdots, X_{r}$ and $Z$ of $\mathrm{g}$ :

$f\left(\left[Z, X_{1}\right], X_{2}, \cdots, X_{r}\right)+f\left(X_{1},\left[Z, X_{2}\right], X_{3}, \cdots, X_{r}\right)+\cdots$

$$
+f\left(X_{1}, \cdots, X_{r-1},\left[Z, X_{r}\right]\right)=0 .
$$

Proof. Let $V$ be the vector space of all $r$-linear functions on $\mathfrak{g}, f \in V$. Then $\operatorname{ad}(\exp t Z)(f)=f$ for all $Z \in \mathfrak{g}$ if and only if ad $Z(f)=0$ for all $Z \in \mathfrak{g}$. Letting $g=\exp t Z$, it follows, from ad $Z=\lim _{t \rightarrow 0}(\operatorname{ad} g-I) / t$, that $(\operatorname{ad} g-I)(f)=0$ for all $Z \in \mathfrak{g}$ if and only if $(\lim (\operatorname{ad} g-I) / t)(f)=0$ for all $Z \in \mathfrak{g}$. The equality below will thus show: $(\operatorname{ad} g-I)\left(f\left(X_{1}, \cdots, X_{r}\right)\right)=0$ for all $X_{i} \in g$ and all $g$ of the form $\exp t Z, Z \in g$, if and only if

$$
f\left(\left[Z, X_{1}\right], X_{2}, \cdots, X_{r}\right)+\cdots+f\left(X_{1}, \cdots, X_{r-1},\left[Z, X_{r}\right]\right)=0
$$

for all $X_{i}$ and $Z$ in g. Since $G$ is connected (hence generated by a neighborhood of the identity element), the proposition is proved:

$$
\begin{aligned}
&(\lim (\operatorname{ad} g-I) / t)\left(f\left(X_{1}, \cdots, X_{r}\right)\right) \\
&= \lim (1 / t)\left(f\left(\operatorname{ad} g X_{1}, \cdots, \operatorname{ad} g X_{r}\right)-f\left(X_{1}, \cdots, X_{r}\right)\right) \\
&= \lim (1 / t)\left(\left(f\left(\operatorname{ad} g X_{1}, \cdots, \operatorname{ad} g X_{r}\right)-f\left(X_{1}, \operatorname{ad} g X_{2}, \cdots, \operatorname{ad} g X_{r}\right)\right)\right. \\
&+\left(f\left(X_{1}, \operatorname{ad} g X_{2}, \cdots, \operatorname{ad} g X_{r}\right)\right. \\
&\left.\left.-f\left(X_{1}, X_{2}, \operatorname{ad} g X_{3}, \cdots, \operatorname{ad} g X_{r}\right)\right)+\cdots\right) \\
&= f\left(\lim \left(\operatorname{ad} g X_{1}-X_{1}\right) / t, \lim \left(\operatorname{ad} g X_{2}\right), \cdots, \lim \left(\operatorname{ad} g X_{r}\right)\right) \\
&+f\left(X_{1}, \lim \left(\operatorname{ad} g X_{2}-X_{2}\right) / t, \lim \left(\operatorname{ad} g X_{3}\right), \cdots\right)+\cdots \\
&= f\left(\left[Z, X_{1}\right], X_{2}, \cdots, X_{r}\right)+f\left(X_{1},\left[Z, X_{2}\right], \cdots, X_{r}\right)+\cdots .
\end{aligned}
$$

2. Connections and curvature [1]. For a detailed discussion, see [1]. All vector fields and all forms will be assumed of class $C^{\infty}$.

Let $M$ be a $C^{\infty}$-manifold, $G$ a Lie group, $g$ the Lie algebra of $G$. Let ( $M, B, G, \pi, \Phi)$ denote the principal bundle with base space $M$, bundle space $B$, group $G$, projection $\pi$ of $B$ onto $M$, and maximal family $\Phi$ of strip maps ( a strip map $\phi$ of $\Phi$ is a 1-1 mapping of some set $\theta \times G$ onto $\pi^{-1} \theta$ - where $\theta$ is an open submanifold of $M$-with $\phi$ and $\phi^{-1}$ both of class $C^{\infty}$.) If $\phi$ is a strip map of $\theta \times G$ onto $\pi^{-1} \theta$ and if $m$ is a point of $\theta$, we denote by $\phi_{m}$ the mapping of $G$ into $B$ which assigns to each $g \in G$ the point $\phi(m, g)$ of $B$. It is assumed that for any two strip maps $\phi, \phi^{\prime}$ for which $\phi_{m}$ and $\phi_{m}^{\prime}$ are both defined, the mapping $\phi_{m}^{-1} \circ \phi_{m}^{\prime}$ is a left-translation by some element of $G$.

A tangent vector $t$ at a point of $B$ is called vertical if $\pi(t)=0$. There is a 
natural mapping $q$ of the elements $X$ of $g$ into vertical vector fields $q X$ on $B$, defined as follows: Let $b$ be any point of $B$, let $m=\pi b$, let $\theta$ be an open submanifold containing $m$, let $\phi$ be a strip map of $\theta \times G$ onto $\pi^{-1} \theta$. Then $b=\phi(m, g)$ for some $g \in G$, and we define $(q X)(b)$ to be the tangent vector $\phi_{m} X(g)$ where $X$ is considered here to be a left-invariant vector field on $G$. Since any two strip maps, when restricted to $\pi^{-1}(m)$, differ only by a left-translation in $G$, this definition is independent of the choice of strip map $\phi$.

Definition. A rule which assigns to each point of $B$ an alternating $r$ linear function from the tangent space at that point into $\mathfrak{g}$, is called a $\mathfrak{g}$ valued $r$-form on $B$.

Definition. A connection on $(M, B, G, \pi, \Phi)$ is a $\mathrm{g}$-valued 1 -form $\omega$ on $B$ which satisfies the following conditions:

(a) if $t$ is a vertical tangent vector at a point $b$ of $B$, then $\omega(t)$ is the unique element $X$ of $g$ with $(q X)(b)=t$;

(b) let $R_{g}$ denote right-translation on the fibres $\pi^{-1}(m)$ of $B$ induced by an element $g$ of $G$, and let $t$ be a tangent vector at a point of $B$; then $\left(R_{\theta}^{*} \omega\right)(t)$ $=\operatorname{ad} g^{-1}(\omega(t))$.

A tangent vector $t$ at a point of $B$ is called horizontal if $\omega(t)=0$. Any tangent vector $t$ at a point of $B$ decomposes into the vector sum of a vertical vector (denoted by $V t$ ) and a horizontal vector (denoted by $H t$ ). This decomposition depends, of course, on the choice of connection $\omega$.

Definition. The covariant derivative $D \Delta$ of an $r$-form $\Delta$ on $B$ is the $(r+1)$ form on $B$ defined by $(D \Delta)\left(t, \cdots, t^{\prime}\right)=d \Delta\left(H t, \cdots, H t^{\prime}\right)$-where $t, \cdots, t^{\prime}$ are $r+1$ tangent vectors at a point of $B$.

Definition. The curvature form $\Omega$ of the connection $\omega$ is the $g$-valued 2-form $D \omega$.

REMARK. Suppose a rule is given which assigns to each point $b$ of $B$ a linear subspace $H(b)$ of the tangent space to $B$ at $b$, and which satisfies the following conditions:

(a) $H(b g)=R_{0} H(b)$, for any $b \in B$ and $g \in G$;

(b) at any point $b$ of $B, H(b)$ is a linear complement to the set of all vertical tangent vectors at $b$;

(c) if $X$ is a $C^{\infty}$ vector field on $B$, then the vector field resulting from projecting $X$ on $H(b)$ at every point $b$, is also $C^{\infty}$.

Then $H$ defines a connection $\omega$ on $B$ in the following way:

(a) if $t$ is a vertical tangent vector at a point $b$ of $B$, then $\omega(t)$ is the unique element $X$ of $g$ with $(q X)(b)=t$.

(b) if $t$ is a tangent vector at a point $b$ of $B$, then $H(b)$ defines a decomposition of $t$ into a vertical part $V t$ and a horizontal part $H t \in H(b)$; and we define $\omega(t)$ to be $\omega(V t)-\omega(V t)$ having already been defined in (a).

We will need the following facts:

(a) If $g \in G$ and $t, t^{\prime}$ are tangent vectors at a point of $B$, then $\left(R_{o}^{*} \Omega\right)\left(t, t^{\prime}\right)$ $=\operatorname{ad} g^{-1}\left(\Omega\left(t, t^{\prime}\right)\right)$. 
(b) If $X, X^{\prime}$ are horizontal vector fields in a neighborhood of a point $b$ of $B$, and if $t, t^{\prime}$ are their values at $b$, then $\Omega\left(t, t^{\prime}\right)=-(1 / 2) \omega\left(\left[X, X^{\prime}\right](b)\right)$.

(c) $D \Omega=0$; this is the Bianchi identity.

(d) If $t, t^{\prime}$ are tangent vectors at a point of $B$, then

$$
d \omega\left(t, t^{\prime}\right)=\Omega\left(t, t^{\prime}\right)-(1 / 2)\left[\omega(t), \omega\left(t^{\prime}\right)\right] ;
$$

this is the equation of structure.

3. The characteristic ring [3]. Let $\omega$ be a connection on the principal bundle $(M, B, G, \pi, \Phi)$, with covariant derivative operator $D$ and curvature form $\Omega$. We now define a mapping of $I(\mathrm{~g})$ into the cohomology ring $H(M)$ of $M$.

Let $r$ be any positive integer and let $T$ be an element of $I^{r}(g)$. We define a real-valued $2 r$-form $\bar{\Omega}_{T}$ on $B$ as follows: If $t_{1}, \cdots, t_{2 r}$ are tangent vectors at a point of $B$, then $\bar{\Omega}_{T}\left(t_{1}, \cdots, t_{2 r}\right)$ is to be the real number Alt $\left(T\left(\Omega\left(t_{1}, t_{2}\right)\right.\right.$, $\left.\cdots, \Omega\left(t_{2 r-1}, t_{2 r}\right)\right)=((2 r) !)^{-1} \Sigma \epsilon T\left(\Omega\left(t_{i_{1}}, t_{i_{2}}\right), \cdots, \Omega\left(t_{i_{2 r-1}}, t_{i_{2 r}}\right)\right)$, where $\epsilon$ is the sign of the permutation taking $1, \cdots, 2 r$ into $i_{1}, \cdots, i_{2 r}$, and where the summation extends over all such permutations.

The form $\bar{\Omega}_{T}$ has three important properties:

(1) It is horizontal - that is, it is zero if one of its arguments is vertical.

(2) $R_{o}^{*} \bar{\Omega}_{T}=\bar{\Omega}_{T}$, for any $g \in G$.

(3) $\bar{\Omega}_{T}$ is closed-that is, $d \bar{\Omega}_{T}=0$.

Proof of (1). $\Omega$ is horizontal.

Proof of (2). Since $R_{0}^{*} \Omega=\operatorname{adg}^{-1} \Omega$, it is clear that $R_{o}^{*} \bar{\Omega}_{T}=\bar{\Omega}\left(\operatorname{adg}^{-1}\right)^{*} T$. This in turn is equal to $\bar{\Omega}_{T}$, since $T$ is invariant under $G$.

Proof of (3). From (1) and (2) it follows that $d \bar{\Omega}_{T}=D \bar{\Omega}_{T}$. Now if we restrict our attention to horizontal forms on $B, D$ is an antiderivation; hence $d \bar{\Omega}_{T}$ $=$ Alt $(T(D \Omega, \Omega, \cdots, \Omega)+\cdots+T(\Omega, \cdots, \Omega, D \Omega))$, which is zero since $D \Omega=0$.

$\bar{\Omega}_{T}$ gives rise in the following way to a real-valued $2 r$-form $\Omega_{T}$ on $M$ : If $x, \cdots, z$ are $2 r$ tangent vectors at a point $m$ of $M$, choose a point $b \in B$ with $\pi b=m$, choose tangent vectors $X, \cdots, Z$ at $b$ with $\pi X=x, \cdots, \pi Z=z$, and define $\Omega_{T}(x, \cdots, z)$ to be the number $\bar{\Omega}_{T}(X, \cdots, Z)$. Since $\bar{\Omega}_{T}$ is horizontal and invariant under right translation by $G$, this definition is independent of the choice of $b$ in $\pi^{-1}(m)$, and of the choice of tangent vectors $X, \cdots, Z$ at $b$ projecting into $x, \cdots, z$ under $\pi$. Furthermore, $d \Omega_{T}=0$ since $d \bar{\Omega}_{T}=0$.

Thus any element $T \in I^{r}(\mathfrak{g})$ defines, in the sense of de Rham, an element of $H^{2 r}(M)$ whose representative is the cocycle $\Omega_{T}$.

Definition. This mapping of $I(\mathfrak{g})$ into $H(M)$ is called the Weil mapping. It is a ring homomorphism. The images of elements of $I^{r}(\mathrm{~g})$ under the Weil mapping are called $2 r$ th characteristic (cohomology) classes of $M$ with respect to the bundle $(M, B, G, \pi, \Phi)$, and the image of $I(\mathfrak{g})$ is called the characteristic ring of $M$ with respect to the bundle $(M, B, G, \pi, \Phi)$.

Note that if $r$ is greater than half the dimension of $M$, the image of $I^{r}(\mathfrak{g})$ 
is zero-for, any differential form on $M$ of degree greater than the dimension of $M$, is the zero-form.

4. The Weil theorem [3]. Let $\alpha$ and $\beta$ be, respectively, $g$-valued $p$ - and $q$-forms on the bundle space $B$ of the principal bundle $(M, B, G, \pi, \Phi)$. We define a $g$-valued $p+q$ form $\alpha \wedge \beta$ on $B$ as follows: If $t_{1}, \cdots, t_{p+q}$ are tangent vectors at a point of $B$, then $(\alpha \wedge \beta)\left(t_{1}, \cdots, t_{p+q}\right)=$ Alt $\left(\left[\alpha\left(t_{1}, \cdots, t_{p}\right)\right.\right.$, $\left.\left.\beta\left(t_{p+1}, \cdots, t_{p+q}\right)\right]\right)$ - the alternation being over the vectors $t_{1}, \cdots, t_{p+q}$, and $[\alpha(), \beta()]$ being the bracket operation in $g$.

It follows from the Jacobi identity that if $\alpha, \beta$ are $\mathfrak{g}$-valued 1 -forms on $B$, then $\alpha \wedge(\alpha \wedge \beta)=(1 / 2)(\alpha \wedge \alpha) \wedge \beta$.

It can also be checked that if $\alpha, \beta$ are, respectively, $g$-valued $p$ - and $q$ forms, then $\beta \wedge \alpha=(-1)^{p q+1} \alpha \wedge \beta$.

Suppose $\omega_{0}$ and $\omega_{1}$ are connections on $(M, B, G, \pi, \Phi)$, with covariant derivative operators $D_{0}$ and $D_{1}$ and curvature forms $\Omega_{0}$ and $\Omega_{1}$; then the equations of structure are $\Omega_{i}=d \omega_{i}+(1 / 2) \omega_{i} \wedge \omega_{i}, i=0,1$. If we define, for $0 \leqq t \leqq 1$, a form $\omega_{t}=t \omega_{1}+(1-t) \omega_{0}$, we get a "homotopy" of $\omega_{t=0}=\omega_{0}$ and $\omega_{t=1}=\omega_{1}$. The form $\omega_{\iota}$ is a connection on $(M, B, G, \pi, \Phi)$ for any such $t$, since both $\omega_{0}$ and $\omega_{1}$ coincide on vertical vectors of $B$ with the inverse of the mapping $q$ defined in $\S 2$; we denote the curvature form of $\omega_{t}$ by $\Omega_{t}$, and we let $u=\omega_{0}-\omega_{1}$.

I. Емма 1. If $\omega$ is a connection with covariant derivative operator $D$, and if $\beta$ is a horizontal $\mathrm{g}$-valued form on $B$ satisfying the rule $R_{0}^{*} \beta=\operatorname{ad}^{-1} \beta$ for all $g \in G$, then $D \beta=d \beta+\omega \wedge \beta$.

Lemma 2. (a) $\Omega_{t}=\Omega_{0}-t D_{0} u+\left(t^{2} / 2\right) u \wedge u$.

(b) $d \Omega_{t} / d t=-\left(D_{0} u-t u \wedge u\right)$.

(c) $D_{0} \Omega_{t}=-\Omega_{t} \wedge u$.

Proof of (a). $u$ is horizontal, hence Lemma 1 shows that $\Omega_{0}-t D_{0} u$ $+\left(t^{2} / 2\right) u \wedge u=d \omega_{0}+(1 / 2) \omega_{0} \wedge \omega_{0}-t d \omega_{0}+t d \omega_{1}-t \omega_{0} \wedge \omega_{0}+t \omega_{0} \wedge \omega_{1}+\left(t^{2} / 2\right) \omega_{0} \wedge \omega_{0}$ $+\left(t^{2} / 2\right) \omega_{1} \wedge \omega_{1}-t^{2} \omega_{0} \wedge \omega_{1}=d \omega_{t}+(1 / 2) \omega_{t} \wedge \omega_{t}=\Omega_{t}$.

Proof of (b). This follows from (a).

Proof of (c). It will be shown below in (1), (2), and (3) that $D_{0}^{2} u=\Omega_{0} \wedge u$, $D_{0}(u \wedge u)=2 D_{0} u \wedge u$, and $(u \wedge u) \wedge u=0$. Hence

$$
\begin{aligned}
D_{0} \Omega_{t} & =0-t D_{0} u+\left(t^{2} / 2\right) D_{0}(u \wedge u) \\
& =-\left(\Omega_{0} u+t^{2} D_{0} u \wedge u-\left(t^{3} / 2\right)(u \wedge u) \wedge u\right. \\
& =-\left(\Omega_{0}-t D_{0} u+\left(t_{2} / 2\right) u \wedge u\right) \wedge t u=-\Omega_{t} \wedge t u .
\end{aligned}
$$

(1) Since $D_{0} u$ is horizontal, Lemma 1 shows that

$$
\begin{aligned}
D_{0}\left(D_{0} u\right) & =d\left(D_{0} u\right)+\omega_{0} \wedge D_{0} u=d\left(d u+\omega_{0} \wedge u\right)+\omega_{0} \wedge\left(d u+\omega_{0} \wedge u\right) \\
& =0+d \omega_{0} \wedge u-\omega_{0} \wedge d u+\omega_{0} \wedge d u+\omega_{0} \wedge\left(\omega_{0} \wedge u\right) \\
& =\left(d \omega_{0}+(1 / 2) \omega_{0} \wedge \omega_{0}\right) \wedge u=\Omega_{0} \wedge u .
\end{aligned}
$$


(2) $u$ is horizontal; hence $D_{0}(u \wedge u)=D_{0} u \wedge u-u \wedge D_{0} u=D_{0} u \wedge u+D_{0} u \wedge u$ $=2 D_{0} u \wedge u$.

(3) $(u \wedge u) \wedge u=(1 / 2) u \wedge(u \wedge u)$, and $(u \wedge u) \wedge u=-u \wedge(u \wedge u)$. Thus $(u \wedge u) \wedge u=-(1 / 2)(u \wedge u) \wedge u$, i.e. $(u \wedge u) \wedge u=0$.

Suppose now that $T$ is an invariant symmetric tensor of $I^{r}(g)$, and that $\Omega_{T}^{0}$ and $\Omega_{T}^{\prime}$ are the characteristic forms on $M$ defined by $T$, and respectively, $\Omega_{0}$ and $\Omega_{1}$. Then we have the following theorem, due to A. Weil:

THEOREM. $\Omega_{T}^{0}$ and $\Omega_{T}^{1}$ are cohomologous on $M$-and hence, the Weil mapping is independent of the choice of connection (and curvature form) on $(M, B, G, \pi, \Phi)$.

Proof. If $A_{1}, \cdots, A_{r}$ are $\mathrm{g}$-valued forms on $B$, we define a real-valued form $\bar{T}\left(A_{1}, \cdots, A_{r}\right)$ on $B$ as follows: $\bar{T}\left(A_{1}, \cdots, A_{r}\right)=$ Alt $\left(T\left(A_{1}, \cdots, A_{r}\right)\right)$, the alternation being over the vector arguments of $A_{1}, \cdots, A_{r}$. We denote $\bar{T}\left(\Omega_{t}, \cdots, \Omega_{t}\right)$ by $\Omega_{T}^{t}$ (this is consistent with the previous definition of $\Omega_{T}^{0}$ and $\Omega_{T}^{l}$.) It will be shown in Lemma 3 that $d \bar{\Omega}_{T}^{t} / d t=-r \cdot d \bar{T}\left(u, \Omega_{t}, \cdots, \Omega_{\imath}\right)$; hence if we define a real-valued form $R(V, X, Y, Z)$ on $B$ by

$$
R(V, X, Y, Z)=-r \int_{0}^{1} \bar{T}\left(V, X-t Y+\left(t^{2} / 2\right) Z, \cdots, X-t Y+\left(t^{2} / 2\right) Z\right) d t
$$

then we have:

$$
\bar{\Omega}_{T}^{1}-\bar{\Omega}_{T}^{0}=\int_{0}^{1}\left(d \bar{\Omega}_{T}^{t} / d t\right) d t=-r \int_{0}^{1} d \bar{T}\left(u, \Omega_{t}, \cdots, \bar{\Omega}_{t}\right) d t .
$$

that is, $\bar{\Omega}_{T}^{1}-\bar{\Omega}_{T}^{0}=d R\left(u, \Omega_{0}, D_{0} u, u \wedge u\right)$.

Clearly, $R\left(u, \Omega_{0}, D_{0} u, u \wedge u\right)$ is a horizontal real-valued form on $B$, invariant under right translation by elements of $G$. Thus $R\left(u, \Omega_{0}, D_{0} u, u \wedge u\right)$ defines a differential form $R$ on $M$ (just as $\bar{\Omega}_{T}$ defined $\Omega_{T}$ ); and so $\Omega_{T}^{1}-\Omega_{T}^{\prime \prime}$ $=d R$, i.e. $\Omega_{T}^{1}$ and $\Omega_{T}^{9}$ are cohomologous on $M$.

Lemma 3. $d \bar{\Omega}_{T} / d t=-r \cdot d \bar{T}\left(u, \Omega_{t}, \cdots, \Omega_{t}\right)$.

Proof. $\bar{T}\left(u, \Omega_{t}, \cdots, \Omega_{t}\right)$ is invariant under right translation by elements of $G$, and is horizontal with respect to $\omega_{0}$; hence $d \bar{T}\left(u, \Omega_{t}, \cdots, \Omega_{t}\right)$ $=D_{0} \bar{T}\left(u, \Omega_{t}, \cdots, \Omega_{t}\right)$, and we have:

$$
\begin{aligned}
d \bar{T}\left(u, \Omega_{t}, \cdots, \Omega_{t}\right)= & D_{0} \bar{T}\left(u, \Omega_{t}, \cdots, \Omega_{t}\right) \\
= & \bar{T}\left(D_{0} u, \Omega_{t}, \cdots, \Omega_{t}\right)-\bar{T}\left(u, D_{0} \Omega_{t}, \Omega_{t}, \cdots, \Omega_{t}\right)-\cdots \\
& -\bar{T}\left(u, \Omega_{t}, \cdots, \Omega_{t}, D_{0} \Omega_{t}\right) \\
= & \bar{T}\left(D_{0} u, \Omega_{t}, \cdots, \Omega_{t}\right)+\bar{T}\left(u, \Omega_{t} \wedge t u, \Omega_{t}, \cdots, \Omega_{t}\right)+\cdots \\
& +\bar{T}\left(u, \Omega_{t}, \cdots, \Omega_{t}, \Omega_{t} \wedge t u\right) \\
= & * \bar{T}\left(D_{0}, u, \Omega_{t}, \cdots, \Omega_{t}\right)-\bar{T}\left(u \wedge t u, \Omega_{t}, \cdots, \Omega_{t}\right) \\
= & \bar{T}\left(D_{0} u-u \wedge t u, \Omega_{t}, \cdots, \Omega_{t}\right)=-\bar{T}\left(d \Omega_{t} / d t, \Omega_{t}, \cdots, \Omega_{t}\right) .
\end{aligned}
$$


The equality* follows from the following extension of the proposition of $\$ 1$ : If $A_{i}(i=1, \cdots, r)$ are $g$-valued $a_{i}$-forms, and if $X$ is a $g$-valued 1 -form (on $B$ ), then the invariance of $T$ under $G$ implies that

$$
\sum(-1)^{a_{1}+\cdots+a_{i-1}} \bar{T}\left(A_{1}, \cdots, A_{i-1}, A_{i} \wedge X, A_{i+1}, \cdots, A_{r}\right)=0 .
$$

Thus we have:

$$
\begin{aligned}
d \bar{\Omega}_{T}^{t} / d t & =\bar{T}\left(d \Omega_{t} / d t, \Omega_{t}, \cdots, \Omega_{t}\right)+\cdots+\bar{T}\left(\Omega_{t}, \cdots, \Omega_{t}, d \Omega_{t} / d t\right) \\
& =r \cdot \bar{T}\left(d \Omega_{t} / d t, \Omega_{t}, \cdots, \Omega_{t}\right)=-r \cdot d \bar{T}\left(u, \Omega_{t}, \cdots, \Omega_{t}\right) .
\end{aligned}
$$

5. Transgressions $[2 ; 3]$.

THEOREM. If $\omega$ is a connection on $(M, B, G, \pi, \Phi)$ with covariant derivative operator $D$ and curvature form $\Omega$; and if $T$ is an element of $I^{r}(g)$; then $\bar{\Omega}_{T}$ $=-d \bar{R}(\omega, \Omega, d \omega, \omega \wedge \omega)$ (the notation being the same as in $\S 4$.)

Proof. The form $\Delta_{t}=\Omega-t d \omega+\left(t^{2} / 2\right) \omega \wedge \omega$ is a "homotopy" of $\Delta_{t=0}=\Omega$ and $\Delta_{t=1}=0$. We have:

$$
\begin{aligned}
d \Delta_{t} & =d \Omega-t d d \omega+\left(t^{2} / 2\right) d(\omega \wedge \omega) \\
& =d \Omega-t d \Omega-(t / 2) d(\omega \wedge \omega)+\left(t^{2} / 2\right) d(\omega \wedge \omega) \\
& =(1-t) d \Omega-(t / 2)(1-t) 2 d \omega \wedge \omega \\
& =(1-t)(D \Omega-\omega \wedge \Omega)-(t / 2)(1-t)(2 d \omega \wedge \omega) \\
& =(1-t) \Omega \wedge \omega-t(1-t) d \omega \wedge \omega+\left(t^{2} / 2\right)(1-t)(\omega \wedge \omega) \wedge \omega \\
& =\left(\Omega-t d \omega+\left(t^{2} / 2\right) \omega \wedge \omega\right) \wedge(1-t) \omega=\Delta_{t} \wedge(1-t) \omega .
\end{aligned}
$$

Thus $d \Delta_{t}=\Delta_{t} \wedge(1-t) \omega$, and so an argument similar to the one used in the proof of the theorem of $\S 4$ shows that

$$
\begin{aligned}
d R(\omega, \Omega, d \omega, \omega \wedge \omega) & =\bar{T}\left(\Delta_{1}, \cdots, \Delta_{1}\right)-\bar{T}\left(\Delta_{0}, \cdots, \Delta_{0}\right) \\
& =0-\bar{T}\left(\Delta_{0}, \cdots, \Delta_{0}\right)=-\bar{\Omega}_{T} .
\end{aligned}
$$

Thus $\bar{\Omega}_{T}$ is a coboundary on $B$, although not on $M$ itself $(R(\omega, \Omega, d \omega, \omega \wedge \omega)$ is not horizontal on $B$, hence does not define a form on $M$ ). If we identify $G$ with a fibre $\pi^{-1}(m)$ of $B$, and denote the inclusion mapping by $i: G \rightarrow B$, then the form $i^{*} R(\omega, \Omega, d \omega, \omega \wedge \omega)$ is closed and hence defines an element of $H(G)$.

Let $A(\mathfrak{g})$ denote the set of all left-invariant differential forms on $g$. Then the above procedure, taking us from $T$ to $\bar{\Omega}_{T}$ and then to $i^{*} R(\omega, \Omega, d \omega, \omega \wedge \omega)$, defines a mapping $\delta$ of $I(g)$ into $A(g)$.

Definition. Any element of $\delta(I(\mathfrak{g}))$-i.e. any $i^{*} R(\omega, \Omega, d \omega, \omega \wedge \omega)$-is called transgressive. Any linear mapping $\lambda$ of $\delta(I(\mathfrak{g}))$ into $I(\mathfrak{g})$ satisfying $\lambda \circ \delta=$ identity, is called a transgression.

6. Special principal bundles. Suppose $M$ is a real orientable Riemannian manifold of dimension $N$. Then the bundle of oriented frames of $M$ is the following principal bundle: 
(1) $G$ is the group $O^{+}(N)$ of all orthogonal real $N \times N$ matrices of determinant 1 .

(2) $B$ is the set of all $(N+1)$-tuples $\left(m, e_{1}, \cdots, e_{N}\right)$, with $m \in M$ and $e_{1}, \cdots, e_{N}$ a positively-oriented orthonormal basis of the tangent space at $m$.

(3) $\pi$ maps the point $\left(m, e_{1}, \cdots, e_{N}\right)$ of $B$ into the point $m$ of $M$.

(4) $\Phi$ consists of all mappings $\phi$ defined as follows: Let $m$ be a point of $M$, let $X_{1}, \cdots, X_{N}$ be vector fields in a neighborhood of $m$ whose values at any point furnish an orthonormal properly-oriented set of tangent vectors at that point, and let $g$ be an element of $G$ (that is, an orthogonal $N \times N$ matrix $\left(g_{i j}\right)$ of determinant 1$)$. Then let $\phi(m, g)$ be the point $\left(m, \sum g_{1 j} X_{j}(m), \cdots\right.$, $\left.\sum g_{N j} X_{j}(m)\right)$ of $B$.

If $M$ is a complex hermitian manifold of complex dimension $N$, then the unitary bundle of $M$ is the following principal bundle:

(1) $G$ is the unitary group $U(N)$.

(2) $B$ is the set of all $(N+1)$-tuples $\left(m, e_{1}, \cdots, e_{N}\right)$, with $m \in M$ and $e_{1}, \cdots, e_{N}$ a complex orthonormal set of tangent vectors at $m$.

(3) $\pi$ and $\Phi$ are defined as in the bundle of oriented frames of a real manifold.

Remark. Suppose $\Omega$ is a curvature form on one of these bundles. Then we can define a function $\tilde{\Omega}$ on $M$ as follows: Let $t, t^{\prime}$ be a pair of tangent vectors at a point $m \in M$. Choose a point $b=\left(m, e_{1}, \cdots, e_{N}\right)$ in the bundle space, and choose a pair of tangent vectors $T, T^{\prime}$ at $b$ with $\pi T=t$ and $\pi T^{\prime}=t^{\prime}$. Then $\widetilde{\Omega}\left(t, t^{\prime}\right)$ is to be the linear transformation on the tangent space at $m$ which, with respect to the basis $e_{1}, \cdots, e_{N}$, has the matrix $\Omega\left(T, T^{\prime}\right)$. We will let $\tilde{\Omega}_{i j}\left(t, t^{\prime}\right)$ denote the $(i, j)$ th entry of this matrix.

7. Some characteristic classes [3]. The bundle of oriented frames of an $N$-dimensional real orientable Riemannian manifold, has as its group the Lie group $O^{+}(N)$; the Lie algebra $g$ of this group is the set of all real skewsymmetric $N \times N$ matrices (matrices $\left(a_{i j}\right)$ satisfying $a_{j i}=-a_{i j}$ ). Consider the tensors $T_{r}(r=1,2, \cdots)$ defined as follows:

$$
T_{r}(A, B, \cdots, E)=\sum \epsilon a_{i_{1} j_{1}} b_{i_{2} j_{2}} \cdots e_{i_{r} j_{r}}
$$

where $A=\left(a_{i j}\right), B=\left(b_{i j}\right), \cdots, E=\left(e_{i j}\right)$ are $r$ skewsymmetric $N \times N$ matrices. (Here, the summation extends over all choices of integers $i_{1}, \cdots, i_{r}$ from among $1, \cdots, N$, and over all permutations $j_{1}, \cdots, j_{r}$ of $i_{1}, \cdots, i_{r} ; \epsilon$ is the sign of the permutation.)

$T_{r}$ is invariant under $O^{+}(N)$, hence gives rise to a characteristic form $\Omega_{T_{r}}$ on $M . \Omega_{T_{r}}$ is called the $2 r$ th Pontrjagin form of $M$. Symbolically, it can be written as $\tilde{\Omega}_{T_{r}}=\sum \epsilon \tilde{\Omega}_{i_{1} j_{1}} \cdots \tilde{\Omega}_{i_{r} j_{r}}$, with $\Omega$ a curvature form on the bundle of oriented frames of $M . \widetilde{\Omega}\left(t, t^{\prime}\right)$ is a skewsymmetric matrix $\left(t, t^{\prime}\right.$ any tangent vectors at a point of $B$ ); hence $\Omega_{T_{r}}$ is zero unless $r$ is even.

Note. If $A$ is any $N \times N$ matrix, the polynomials $p_{r}(A)$ of the expansion $\operatorname{det}(\lambda I-A)=\lambda^{N}+p_{1}(A) \lambda^{N-1}+\cdots+p_{N}(A)$ are: 


$$
p_{r}(A)=(-1)^{r} \sum \epsilon a_{i_{1} j_{1}} a_{i_{2} j_{2}} \cdots a_{i_{r} j_{r}} .
$$

Thus $T_{r}(A, \cdots, A)=(-1)^{r} \cdot p_{r}(A)$.

Suppose now that $M$ has even dimension $2 n$. The tensor $S$ which assigns to any $n$ skewsymmetric $2 n \times 2 n$ matrices $A, B, \cdots, H$ the value

$$
S(A, B, \cdots, H)=\sum \epsilon a_{i_{1} i_{2}} b_{i_{3} i_{4}} \cdots h_{i_{2 n-1} i_{2 n}}
$$

is invariant under $\mathrm{O}^{+}(2 n)$ (here $\epsilon$ is the sign of the permutation taking $1, \cdots, 2 n$ into $i_{1}, \cdots, i_{2 n}$, and the summation extends over all such permutations). Thus $S$ gives rise to a characteristic form $\Omega_{S}$ on $M$, called the Euler-Poincaré form of $M$. Symbolically, it can be written as $\Omega_{S}$ $=\sum \epsilon \tilde{\Omega}_{i_{1} i_{2}} \cdots \tilde{\Omega}_{i_{2 n-1} i_{2 n}}$.

If $M$ has odd dimension, we define the Euler-Poincare form of $M$ to be the zero-form on $M$.

A proof of the following theorem can be found in [3].

The Gauss-Bonnet theorem. If $M$ is a compact orientable Riemannian manifold, then $\int_{M} \Omega_{X}$ is equal to the Euler-Poincaré characteristic of $M .\left(\Omega_{X}\right.$ is defined to be $\Omega_{S}$ if $M$ has odd dimension, and $(-1)^{n} / n !(4 \pi) n \cdot \Omega_{S}$ if $M$ has even dimension $2 n$.)

$N o t e$. If $A$ is a real skewsymmetric $2 N \times 2 N$ matrix, then $(S(A, \cdots, A))^{2}$ $=4^{N} \cdot \operatorname{det} A$.

Finally, suppose $M^{\prime}$ is a complex hermitian manifold of complex dimension $N^{\prime}$. We consider its unitary bundle. The group of this bundle is $U\left(N^{\prime}\right)$, and the Lie algebra $g$ of $U\left(N^{\prime}\right)$ is the set of all $N^{\prime} \times N^{\prime}$ skew hermitian matrices (complex matrices $\left(a_{i j}\right)$ satisfying $a_{j i}=-\bar{a}_{i j}$ ). Each tensor $T_{r}(A, B, \cdots, E$ ) $=\sum \epsilon a_{i_{1} j_{1}} b_{i_{2} j_{2}} \cdots e_{i_{r} j_{r}}$ is an invariant symmetric tensor on $\mathfrak{g}$ (here $A, B, \cdots, E$ are $r$ elements of $\mathfrak{g}$ ), and so $T$, defines a characteristic form $\Omega_{T_{r}}$ on $M^{\prime} . \Omega_{T_{r}}$ is called the $2 r$ th Chern form of $M^{\prime}$. Symbolically, it can be written as $\Omega_{T_{r}}=\sum \epsilon{\tilde{\Omega_{i}, 1_{1}}}^{\prime} \cdots \tilde{\Omega}_{t_{r} \gamma_{r}}^{\prime}$, with $\Omega^{\prime}$ a curvature form on the unitary bundle of $M^{\prime}$.

8. Semi-simple Lie algebras [4]. From now on, $i$ will denote the squareroot of -1 .

The fundamental bilinear form of a Lie algebra $g$ is the form $(X, Y)$ $=$ trace $(\operatorname{ad} X \circ$ ad $Y), X, Y \in \mathfrak{g} . \mathfrak{g}$ is called semi-simple if the fundamental bilinear form is nondegenerate on $\mathrm{g}$. It is known that the Lie algebra of a compact Lie group is always the algebraic direct sum of a semi-simple Lie algebra and an abelian Lie algebra.

The rank of a compact Lie group $G$ is the dimension of any maximal abelian subgroup of $G$.

Suppose $G$ is a compact semi-simple Lie group (that is, the Lie algebra $\mathfrak{g}$ of $G$ is semi-simple), and suppose $H(\mathfrak{g})$ is a maximal abelian subalgebra of $\mathfrak{g}$. So the dimension of $H(\mathfrak{g})$ is the rank of $G$. Let $\mathrm{g}^{\prime}$ denote the complexification 
$\mathfrak{g}+i \mathfrak{g}$ of $\mathfrak{g}$, and let $H\left(\mathfrak{g}^{\prime}\right)$ denote the complexification $H(\mathfrak{g})+i H(\mathfrak{g})$ of $H(\mathfrak{g})$. Then $H\left(g^{\prime}\right)$ is a maximal abelian subalgebra of $g^{\prime}$, and $\mathfrak{g}^{\prime}$ has a vector-space decomposition $\mathfrak{g}^{\prime}=\sum \mathfrak{g}_{\alpha}$ satisfying:

(a) $\alpha$ is a complex-valued linear function (called a root) on $H\left(\mathfrak{g}^{\prime}\right)$, and $\mathfrak{g}_{\alpha}$ is the set of all eigenvectors of $\alpha:[H, X]=\alpha(H) X$ for any $H \in H\left(\mathfrak{g}^{\prime}\right)$ and $X \in \mathfrak{g}_{\alpha}$.

(b) $g_{0}=H\left(\mathfrak{g}^{\prime}\right)$.

(c) Each $g_{\alpha}$ is one-dimensional.

(d) If $\alpha, \beta$, and $\alpha+\beta$ are roots, then $\left[g_{\alpha}, g_{\beta}\right]=g_{\alpha+\beta}$; if $\alpha+\beta$ is not a root, then $\left[\mathfrak{g}_{\alpha}, \mathfrak{g}_{\beta}\right]=0$.

(e) If $\alpha$ is a root, then $k \alpha$ is a root if and only if $k=0,1$, or -1 .

$H\left(\mathfrak{g}^{\prime}\right)$ is called a Cartan subalgebra of $\mathfrak{g}^{\prime}$; an element of $g_{\alpha}$ is called a root vector of $\mathfrak{g}^{\prime}$ with respect to $H\left(\mathfrak{g}^{\prime}\right)$.

The following relations hold: $\left(H, \mathfrak{g}_{\alpha}\right)=0,\left(\mathfrak{g}_{\alpha}, \mathfrak{g}_{\beta}\right)=0$, and $(H, H) \neq 0$, for any $H \in H\left(\mathfrak{g}^{\prime}\right)$ and any roots $\alpha, \beta$ with $\beta \neq-\alpha$. It is possible to choose one element $e_{\alpha}$ from each $g_{\alpha}$ in such a way that $\left(e_{\alpha}, e_{-\alpha}\right)=-1$, and that the numbers $N_{\alpha \beta}$ defined by $\left[e_{\alpha}, e_{\beta}\right]=N_{\alpha \beta} e_{\alpha+\beta}$ satisfy the relations: (1) $N_{-\alpha,-\beta}=N_{\alpha \beta}$ (2) $N_{\beta \alpha}=-N_{\alpha \beta}$ (3) $N_{\alpha \beta}^{2}$ is a non-negative rational number.

From now on, it will be assumed that the $e_{\alpha}$ 's have been chosen in this manner. We then define, for each root $\alpha$, elements $X_{\alpha}, Y_{\alpha}, H^{\alpha}, \bar{H}^{\alpha}$ of $\mathfrak{g}^{\prime}$ :

$$
\begin{array}{ll}
X_{\alpha}=e_{\alpha}+e_{-\alpha}, & H^{\alpha}=-\left[e_{\alpha}, e_{-\alpha}\right], \\
Y_{\alpha}=i\left(e_{\alpha}-e_{-\alpha}\right), & \bar{H}^{\alpha}=i H^{\alpha} .
\end{array}
$$

The vectors $X_{\alpha}, Y_{\alpha}$ are called quasi-root vectors of $\mathfrak{g}^{\prime}$. The following facts are known.

(a) The elements $X_{\alpha}, Y_{\alpha}, \bar{H}^{\alpha}$ span $\mathfrak{g}$ and lie in $\mathfrak{g}$. So a vector $\sum a_{\alpha} e_{\alpha}$ $+\sum b_{\alpha} H^{\alpha}$ of $\mathfrak{g}^{\prime}\left(a_{\alpha}, b_{\alpha}\right.$ complex numbers $)$ is in $\mathfrak{g}$ if and only if each $b_{\alpha}$ is pure imaginary and $a_{-\alpha}=\bar{a}_{\alpha}$ (complex conjugate).

(b) If $\alpha$ is a root, $H \in H\left(\mathrm{~g}^{\prime}\right)$, and $h \in H(\mathrm{~g})$, then $\left(H^{\alpha}, H\right)=\alpha(H), \alpha\left(H^{\alpha}\right)$ is a positive real number, and $\alpha(h)$ is pure imaginary.

It can easily be seen that for any $H \in H\left(\mathfrak{g}^{\prime}\right)$ and any root $\alpha,\left[H, X_{\alpha}\right]$ $=-i \alpha(H) Y_{\alpha}$ and $\left[H, Y_{\alpha}\right]=i \alpha(H) X_{\alpha}$.

From now on, we will be dealing only with compact semi-simple Lie groups; this serves to simplify the notation. All results hold, however, for compact Lie groups, since every compact Lie group has a decomposition of its Lie algebra into the algebraic direct sum of an abelian and a semi-simple Lie algebra. Only slight modifications of the proofs are needed.

9. Homogeneous spaces [6]. Hereafter, $G$ will denote a compact semisimple Lie group, and $K$ a closed connected subgroup of $G$. The Lie algebras of $G$ and $K$ will be denoted by $g$ and $\mathfrak{l}$ respectively, and $\mathfrak{m}$ will denote the set of all $X \in \mathfrak{g}$ for which $(X, k)=0$, all $k \in \mathfrak{l}$. Then ad $K$ is a set of linear transformations of $m$ (and so [ $m, \mathfrak{l}]$ lies in $m$ ), and $\mathfrak{g}$ is the vector-space direct sum of $\mathfrak{m}$ and $\mathfrak{t}$. 
We can associate $g$ with $G_{e}$ (the tangent space to $G$ at the identity $e$ ). The fundamental bilinear form thus can be considered to be on $G_{e}$, and is invariant under ad $G$. If we define a bilinear form on the tangent spaces at other points of $G$ by right-translating the fundamental bilinear form at $e$, the resulting bilinear form on $G$ will be both left and right invariant under $G$. It induces a metric on $G / K$ in the natural way (see [6]).

We can also associate $m$ with the tangent space $(G / K)_{e K}$ to $G / K$ at the point $e K$. Then $K$ induces a group of orientation-preserving isometries of $(G / K)_{e K}$, and in fact the effect of any ad $k$ on $m(k \in K)$ is the same as the effect of left-translation by $k$ on $(G / K)_{e K}$.

If $t$ is a tangent vector at a point of $G$, we define an element $t_{\mathfrak{f}}$ of $\mathfrak{g}$ as follows: Extend $t$ to a left-invariant vector field on $G$, thus defining an element of $g ; t$ is to be the projection of this element on $t$.

10. The canonical connection of the second kind. There are now two principal bundles to be considered: The bundle $\left(G / K, B, O^{+}(N), \pi, \Phi\right)$ of oriented frames of $G / K$ (here $N$ denotes the dimension of $G / K$ ), and the coset bundle $\left(G / K, G, K, p, \Phi^{\prime}\right)$-where $p$ is the natural projection of $G$ onto $G / K$, and where the strip maps $\Phi^{\prime}$ are the natural ones.

Let $\omega$ be the connection on the coset bundle of $G / K$ defined as follows: If $t$ is a tangent vector at a point of $G$, then $\omega(t)=t_{t}$. This choice of connection makes horizontal the elements of $G_{e}$ corresponding to $\mathrm{m}$; and so if $\Omega$ is the curvature form of $\omega$ and if $X, Y$ are tangent vectors on $G$ which, when extended left-invariantly, generate elements of $\mathfrak{m}$, then

$$
\Omega(X, Y)=-(1 / 2)[X, Y]_{t} .
$$

The connection $\omega$ induces a connection on the bundle of oriented frames of $G / K$ in the following way:

A. Let $X_{1}, \cdots, X_{N}$ be horizontal left-invariant vector fields on $G$ which, at any point of $G$, define a set of orthonormal tangent vectors whose orientation is consistent with that of $G / K$. Then there is a mapping $\Delta$ of $G$ into $B$ : $\Delta(g)=\left(g K, p X_{1}(g), \cdots, p X_{N}(g)\right) . \Delta(g)$ can be interpreted as the left-action of $g$ on $(G / K)_{e K} ; \Delta$ maps $K$ into $O^{+}(N)$ by taking any element $k$ of $K$ into the matrix of ad $k$ acting on the vectors $X_{1}(e), \cdots, X_{N}(e)$-i.e. acting on $\mathfrak{m}$. Clearly, $\pi \circ \Delta=p$.

B. $\Delta$ can be used to define a connection in $B . H(\Delta(g))$ is to be the image under $\Delta$ of the space of horizontal tangent vectors (with respect to $\omega$ ) to $G$ at $g$, and $H$ at other points of $\pi^{-1}(g K)$ is to be defined by the relations $H(b o)$ $=R_{0} H(b), b \in B$ and $o \in O^{+}(N)$. It is clear that this $H$ is a connection on the bundle of oriented frames of $G / K$; let ad $\omega$ denote the corresponding 1-form on $B$ with values in the Lie algebra of $O^{+}(N)$, and let ad $\Omega$ denote the curvature form of ad $\omega$. ad $\omega$ is called the canonical connection of the second kind on $G / K$.

C. We have the following simple results: 
(a) If $t$ is a vertical tangent vector at a point $g \in G$, then $\Delta(t)$ is a vertical tangent vector at $\Delta(g)$, with $(\operatorname{ad} \omega)(\Delta(t))=\Delta(\omega(t))$. Also, $\Delta(\omega(t))$ is the matrix of ad $t_{t}$ with respect to the basis $X_{1}, \cdots, X_{N}$ of $\mathrm{m}$.

(b) If $x, y$ are horizontal tangent vectors at a point $\Delta(g)$, then there exist horizontal vector fields $X, Y$ at $g$ with $\Delta(X), \Delta(Y)$ horizontal vector fields whose values at $\Delta(g)$ are $x, y$.

Proposition 1. If $x, y, X, Y$ are as above, then

$$
(\operatorname{ad} \Omega)(x, y)=\operatorname{ad}(\Omega(X(g), Y(g)))[\mathfrak{m}]
$$

(the symbol ad $Z[\mathfrak{m}], Z \in \mathfrak{l}$, denotes the matrix of $\operatorname{ad} Z$ with respect to the basis $X_{1}, \cdots, X_{N}$ of $\left.\mathfrak{m}\right)$.

Proof. $(\operatorname{ad} \Omega)(x, y)=-\operatorname{ad} \omega([\Delta(X), \quad \Delta(Y)](\Delta(g)))=-\Delta(\omega[X, \quad Y](g))$ $=\Delta(\Omega(X(g), Y(g)))$.

Now ad $\Omega$ induces the form ad $\Omega$ on $G / K$. Let $g$ be a point of $G$. We will denote $p X_{j}$ by $Y_{j}$. Then $L_{g} Y_{j}(e)=Y_{j}(g)$-here $L_{g}$ denotes left-translation by $g$-and $(\mathrm{a} \widetilde{\mathrm{d}} \Omega)\left(L_{0} Y_{j}(e), L_{0} Y_{k}(e)\right)$ is the linear transformation whose matrix with respect to the basis $L_{g} Y_{r}(e)$ is $(\operatorname{ad} \Omega)\left(\Delta\left(Y_{j}(g)\right), \Delta\left(Y_{k}(g)\right)\right)=-\Delta\left(\omega\left[Y_{j}\right.\right.$, $\left.\left.Y_{k}\right](g)\right)$. Similarly, $(\mathrm{a} \tilde{\mathrm{d}} \Omega)\left(Y_{j}(e), Y_{k}(e)\right)$ is the linear transformation whose matrix with respect to the basis $Y_{r}(e)$ is $-\Delta\left(\omega\left[Y_{j}, Y_{k}\right](e)\right)$. Thus, since a $\Omega$ is bilinear, we have:

Proposition 2. If $t, t^{\prime}$ are tangent vectors at $e K$ and if $g \in G$, then $(\mathrm{a} \widetilde{\mathrm{d}} \Omega)\left(L_{\theta} t, L_{\theta} t^{\prime}\right)$ w.r.t. $\left\{L_{o} Y_{r}(e)\right\}=(\mathrm{a} \widetilde{\mathrm{d}} \Omega)\left(t, t^{\prime}\right)$ w.r.t. $\left\{Y_{r}(e)\right\}$.

Proposition 2 shows that one need consider characteristic forms on $G / K$ (with respect to the bundle of oriented frames) only on vectors at $e K$ : For, if one uses the connection ad $\omega$, then ad $\Omega$ at any point $g K$ can be expressed in terms of ad $\Omega$ at $e K$.

Proposition 3. Let $c(K)$ and $c\left(O^{+}\right)$denote respectively the characteristic rings of $G / K$ with respect to the coset bundle and the bundle of oriented frames. Then $c\left(O^{+}\right)$is contained in $c(K)$.

Proof. We use the connections $\omega$ and ad $\omega$. Let $\Omega_{T}$ be a form of $c\left(O^{+}\right)$, and define a tensor $\bar{T}$ on by: $\bar{T}(X, \cdots, Z)=T(\operatorname{ad} X[\mathfrak{m}], \cdots$, ad $Z[\mathfrak{m}])$, $X, \cdots, Z \in \mathfrak{l}$. It follows from Proposition 1 that $\Omega_{\tilde{T}}$ (using the curvature form $\Omega$ ) is identical with $\Omega_{T}$ (using the curvature form ad $\Omega$ ). Since $\Omega_{T}$ is an element of $c(K)$ if $\bar{T}$ is an invariant symmetric tensor on $\mathfrak{l}$, it remains to show that $\bar{T}$ is invariant (clearly, it is symmetric): So suppose $X, Y, \cdots, Z$ and $A$ are elements of $\mathfrak{t}$; then:

$$
\begin{aligned}
\bar{T}([A, X], Y, \cdots, & Z)+\cdots+\bar{T}(X, Y, \cdots,[A, Z]) \\
& =T(\operatorname{ad}[A, X][\mathfrak{m}], \text { ad } Y[\mathfrak{m}], \cdots, \text { ad } Z[\mathfrak{m}])+\cdots \\
& =T([\operatorname{ad} A[\mathfrak{m}], \text { ad } X[\mathfrak{m}]], \text { ad } Y[\mathfrak{m}], \cdots, \text { ad } Z[\mathfrak{m}])+\cdots
\end{aligned}
$$


and this final sum is zero since $T$ is invariant under $O^{+}(N)$ and since ad $K$ [m] is a subset of $O^{+}(N)$. Since $K$ is connected, it follows that $\bar{T}$ is invariant under $K$.

Proposition 4 (See $[2$, p. 70]). Let $J$ be the set of all elements of $I(\mathfrak{l})$ which can be written in the form $\sum_{i} T_{i} S_{i}$, where $T_{i}$ and $S_{i}$ are elements of $I(\mathfrak{l})$ of degree greater than zero, and where each $S_{i}$ can be extended to an element of $I(g)$. Then a characteristic form $\Omega_{T}$ of $c(K)$ is cohomologous to zero on $G / K$ if and only if $T$ is an element of $J$.

Proof. We will prove here only that every $T \in J$ gives rise to a form $\Omega_{T}$ which is cohomologous to zero on $G / K$; the other half of the proposition will not be needed here, and its proof is considerably more difficult.

So suppose $T=\sum T_{i} S_{i}$, with each $S_{i}$ extendable to an element of $I(\mathfrak{g})$. It will be shown below that each $\Omega_{S_{i}}$ is cohomologous to zero-that is, $\Omega_{S_{i}}=d \Delta_{i}$, for some form $\Delta_{i}$ on $G / K$. Then, since the Weil mapping is a ring homomorphism, and since $d \Omega_{T_{i}}=0$, it follows that $\Omega_{T}=d\left(\sum \Omega_{T_{i}} \wedge \Delta_{i}\right)$.

Suppose then that $S$ is an element of $I(\mathfrak{t})$, extendable to an element of $I(g)$. The principal bundle with fibre $G$ associated with the principal bundle $(G / K, G, K, p)$, is the bundle $\left(G / K, G \times{ }_{K} G, G, p^{\prime}\right)$, where $G \times{ }_{K} G$ is the set of equivalence classes of $G \times G$ under the equivalence relation $g k \times g^{\prime}=g \times k^{-1} g^{\prime}$. Let $\omega$ be the connection on $(G / K, G, K, p)$ defined previously, and let $\Omega$ be its curvature form. An argument similar to the one used in the proof of Proposition 1, above, shows that $\omega$ and $\Omega$, and the natural mapping of $G$ into $G \times{ }_{K} G$, give rise to a connection $\omega^{\prime}$ on $\left(G / K, G \times{ }_{K} G, G, p^{\prime}\right)$, with curvature form $\Omega^{\prime}$, satisfying: $S(\Omega, \cdots, \Omega)=S\left(\Omega^{\prime}, \cdots, \Omega^{\prime}\right.$ ) (both of these forms are considered to be on the base space $G / K$.)

Now $S \in I(\mathfrak{g})$, hence $S\left(\Omega^{\prime}, \cdots, \Omega^{\prime}\right)$ is a characteristic form of $G / K$ with respect to the bundle $\left(G / K, G \times{ }_{K} G, G, p^{\prime}\right)$. Since the Weil mapping is independent of the choice of connection, and since this bundle is trivial $(f(g K)$ $=g \times g^{-1}$ is a cross-section), it follows that $S\left(\Omega^{\prime}, \cdots, \Omega^{\prime}\right)$ is cohomologous to zero on $G / K$, hence that $\Omega_{S}=S(\Omega, \cdots, \Omega)$ is cohomologous to zero on $G / K$.

Proposition 5. Let $H(\mathfrak{t})$ be a maximal abelian subalgebra of $\mathfrak{t}$, let $\mathfrak{g}^{\prime}, \mathfrak{t}^{\prime}, \mathfrak{m}^{\prime}$, and $H\left(\mathfrak{l}^{\prime}\right)$ denote respectively the complexifications of $\mathfrak{g}, \mathfrak{l}, \mathfrak{m}$, and $H(\mathfrak{l})$; so $H\left(\mathfrak{l}^{\prime}\right)$ is a maximal abelian subalgebra of $\mathfrak{f}^{\prime}$. Extend $H\left(\mathfrak{f}^{\prime}\right)$ to a maximal abelian subalgebra $H\left(\mathfrak{g}^{\prime}\right)$ of $\mathfrak{g}^{\prime}$. Let $H\left(\mathfrak{m}^{\prime}\right)=H\left(\mathfrak{g}^{\prime}\right) \cap \mathfrak{m}^{\prime}$. Then $H\left(\mathfrak{g}^{\prime}\right)=H\left(\mathfrak{l}^{\prime}\right)+H\left(\mathfrak{m}^{\prime}\right)$.

Proof. Suppose $H \in H\left(\mathfrak{g}^{\prime}\right)$, with $H=k+m \quad\left(k \in \mathfrak{f}^{\prime}, \quad m \in \mathfrak{m}^{\prime}\right)$. Then $0=\left[H, H\left(\mathfrak{l}^{\prime}\right)\right]=\left[k, H\left(\mathfrak{l}^{\prime}\right)\right]+\left[m, H\left(\mathfrak{l}^{\prime}\right)\right]$. Since $\left[k, H\left(\mathfrak{l}^{\prime}\right)\right] \in \mathfrak{l}^{\prime}$ and $\left[m, H\left(\mathfrak{l}^{\prime}\right)\right]$ $\in \mathfrak{m}^{\prime}$, it follows that $\left[k, H\left(\mathfrak{l}^{\prime}\right)\right]=0$. But $H\left(\mathfrak{l}^{\prime}\right)$ is a maximal abelian subalgebra of $\mathfrak{l}^{\prime}$; hence $k \in H\left(\mathfrak{l}^{\prime}\right)$, and $m=H-k \in \mathfrak{m}^{\prime} \cap H\left(\mathfrak{g}^{\prime}\right)=H\left(\mathfrak{m}^{\prime}\right)$. Thus $H \in H\left(\mathfrak{l}^{\prime}\right)$ $+H\left(\mathfrak{m}^{\prime}\right)$.

\section{The abelian case.}

Theorem. If $K$ is abelian, all Pontrjagin forms of $G / K$ are cohomologous to zero. 
Proof. Let $N, N^{\prime}$ denote the dimensions of $G / K, G$. Since $K$ is abelian, $\mathfrak{l}$ lies in $H(\mathfrak{g})$. If we choose an orthonormal basis of $g$ whose first $N$ elements are an orthonormal properly-oriented basis of $m$, then with respect to this basis we have

$$
\operatorname{ad} H[\mathfrak{g}]=\left(\frac{\operatorname{ad} H[\mathfrak{m}]}{0} \mid \frac{0}{0}\right)
$$

for any element $H$ of $\mathfrak{t}$.

Define $T, T^{\prime}$ as follows:

(a) If $A=\left(a_{i j}\right), B=\left(b_{i j}\right), \cdots, E=\left(e_{i j}\right)$ are $r$ skewsymmetric real $N \times N$ matrices, then $T(A, B, \cdots, E)=\sum \epsilon a_{i_{1} j_{1}} b_{i_{2} j_{2}} \cdots e_{i_{r} j_{r}}\left(i_{1}, \cdots, i_{r}\right.$ chosen from among the integers $1, \cdots, N)$.

(b) If $A^{\prime}=\left(a_{i j}^{\prime}\right), B^{\prime}=\left(b_{i j}^{\prime}\right), \cdots, E^{\prime}=\left(e_{i j}^{\prime}\right)$ are $r$ skewsymmetric real $N^{\prime} \times N^{\prime}$ matrices, then $T^{\prime}\left(A^{\prime}, B^{\prime}, \cdots, E^{\prime}\right)=\sum \epsilon a_{i_{1} j_{1}}^{\prime} b_{i_{2} j_{2}}^{\prime} \cdots e_{i_{r} j_{r}}^{\prime}\left(i_{1}, \cdots, i_{r}\right.$ chosen from among the integers $\left.1, \cdots, N^{\prime}\right)$.

Clearly $T$ and $T^{\prime}$ are symmetric and are invariant under $O^{+}(N)$ and $\mathrm{O}^{+}\left(N^{\prime}\right)$ respectively. They define tensors $\bar{T}, \bar{T}^{\prime}$ on $\mathfrak{t}$ in the following way: If $H_{1}, \cdots, H_{r}$ are $r$ elements of $\mathfrak{t}$, then $\bar{T}\left(H_{1}, \cdots, H_{r}\right)=T\left(\operatorname{ad} H_{1}[\mathfrak{m}], \cdots\right.$, ad $\left.H_{r}[\mathfrak{m}]\right)$ and $\bar{T}^{\prime}\left(H_{1}, \cdots, H_{r}\right)=T^{\prime}\left(\operatorname{ad} H_{1}[\mathfrak{g}], \cdots\right.$, ad $\left.H_{r}[\mathfrak{g}]\right)$. Since $K$ is connected, $\bar{T}$ and $\bar{T}^{\prime}$ are elements of $I(\mathfrak{l})$; and from the expression given above for any matrix ad $H[\mathfrak{g}](H \in \mathfrak{l})$ it follows that the summands of $\bar{T}^{\prime}$ give zero contributions except in the cases when all the indices $i_{1}, \cdots, i_{r}$ lie between 1 and $N$, hence that $\bar{T}$ and $\bar{T}^{\prime}$ coincide on $\mathfrak{t}$. The method of proof of Proposition $3, \S 10$, shows that the $2 r$ th Pontrjagin characteristic form of $G / K$ is $\Omega_{\bar{T}}$; and so the $2 r$ th Pontrjagin form of $G / K$ is $\Omega_{\bar{T}^{\prime}}$.

If the tensor $\bar{T}^{\prime \prime}$ on $\mathrm{g}$ is defined by $\bar{T}^{\prime \prime}(X, \cdots, Z)=T^{\prime}(\operatorname{ad} X[\mathrm{~g}], \cdots$, ad $Z[\mathfrak{g}]), X, \cdots, Z r$ elements of $\mathfrak{g}$, then $\bar{T}^{\prime \prime}$ is an element of $I(\mathfrak{g})$ and also an extension of $\bar{T}^{\prime}$ to all of $\mathrm{g}$. Thus $\bar{T}^{\prime} \in J$ (see Proposition $4, \S 10$ ), and hence $\Omega_{\overline{T^{\prime}}}$ is cohomologous to zero.

12. Complex and symmetric homogeneous spaces $[6 ; 8]$. A homogeneous space $G / K$ ( $G$ a compact connected Lie group, $K$ a closed connected subgroup of $G$ ) is called symmetric if there is an involutive automorphism $\Delta$ of $G$ (that is, $\Delta^{2}=$ identity) for which $K$ lies between the set $K_{\Delta}$ of all fixed points of $\Delta$ and the identity component of $K_{\Delta} . \Delta$ induces an involutive automorphism of $\mathfrak{g}$ (again denoted by $\Delta$ ); if $\overline{\mathfrak{m}}$ denotes the eigenspace for the eigenvalue -1 of $\Delta$, then $\mathfrak{g}=\mathfrak{l}+\overline{\mathfrak{m}}$, and $\Delta([\overline{\mathfrak{m}}, \overline{\mathfrak{m}}])=[\Delta \overline{\mathfrak{m}}, \Delta \overline{\mathfrak{m}}]=[-\overline{\mathfrak{m}},-\overline{\mathfrak{m}}]=[\overline{\mathfrak{m}}, \overline{\mathfrak{m}}]$, i.e. $[\overline{\mathfrak{m}}, \overline{\mathfrak{m}}]$ lies in $\mathfrak{l}$. It is easily seen that $\overline{\mathfrak{m}}$ is the orthogonal complement of $\mathfrak{l}$ with respect to the fundamental bilinear form of $\mathfrak{g}$. So we conclude that $[\mathfrak{m}, \mathfrak{m}] \subseteq \mathfrak{f}$.

By a $C$-space we will mean an even-dimensional homogeneous space $G / K$, with $G$ a compact semi-simple Lie group and $K$ a closed connected subgroup of $G$ whose semi-simple part coincides with the semi-simple part of the centralizer of a toral subgroup of $G$. Wang has shown in [8] that the $C$-spaces are exactly the simply-connected compact complex homogeneous manifolds. 
TheOREM. If $G / K$ is a symmetric space or a $C$-space, then all characteristic $c(K)$ r and $c\left(O^{+}\right)$-forms of degree greater than (dimension $\left.G / K\right)-($ rank $G$ -rank $K)$, are zero. If $G / K$ is a symmetric $C$-space, then rank $G=\operatorname{rank} K$.

Proof. If $G / K$ is symmetric, it has been proved by H. Cartan [2] that the cohomology ring $H(G / K)$ of $G / K$ is ring-isomorphic with a tensor product $c(K) \otimes Y$, where $Y$ is the Grassman algebra over a vector space of dimension $N-R(N=\operatorname{dim} G / K, R=\operatorname{rank} G-\operatorname{rank} K)$ and where the degree of an element $c \otimes y(c \in c(K), y \in Y)$ is understood to be the sum of the degrees of $c$ and $y$ if these are both homogeneous elements. So if $\Omega_{T}$ were a nonzero $c(K)$-form of degree greater than $N-R$, and if $a_{1}, \cdots, a_{N-R}$ were a basis of the vector space generating $Y$, the element $\Omega_{T} \otimes\left(a_{1} \cdots a_{N-R}\right)$ would be a nonzero element of $H(G / K)$ of degree greater than $N$. This cannot be, since $N$ is the dimension of $G / K$. It follows that all $c(K)$-forms of degree greater than $N-R$ are zero; hence all $c\left(O^{+}\right)$-forms of degree greater than $N-R$ are zero, since $c\left(O^{+}\right)$is contained in $c(K)$.

Next, suppose $G / K$ is a $C$-space. It will now be shown that every pair of root vectors $e_{\alpha}, e_{-\alpha}$ lies either in $\mathfrak{t}^{\prime}$ or in $\mathfrak{m}^{\prime}$, hence that every pair of quasiroot vectors $X_{\alpha}, Y_{\alpha}$ lies either in $\mathfrak{l}$ or in $\mathrm{m}$. Since $\left[H, X_{\alpha}\right]=-i \alpha(H) Y_{\alpha}$ and $\left[H, Y_{\alpha}\right]=i \alpha(H) X_{\alpha}$ for any $H \in H\left(\mathfrak{g}^{\prime}\right)$, it follows from this that $[H(\mathfrak{m}), \mathfrak{m}] \mathfrak{l}=0$ (where $H(\mathfrak{m})=\mathfrak{m} \cap H\left(\mathfrak{m}^{\prime}\right)$ ). Thus $\Omega(H, X)=0$ for any $H \in H(\mathfrak{m})$ and $X \in \mathfrak{m}$. But then suppose $\Omega_{T}$ is a $c(K)$-form of degree greater than $N-R$. The dimension of $H(\mathrm{~m})$ is $R$, and so we can choose a basis of $\mathfrak{n t}$ whose first $R$ elements are in $H(\mathrm{~m})$. Consider any term $\Omega_{T}(X, \cdots, Z)$, where $X, \cdots, Z$ are elements of $m$. If this term is expanded in terms of the above basis of $m$ (that is, $X, \cdots, Z$ are written in terms of this basis), then every term $\Omega_{T}(\cdots)$ of this expansion will have at least one entry from $H(\mathfrak{m})$. Since $\Omega(H(\mathfrak{m}), \mathfrak{m})$ is zero, it follows that each $\Omega_{T}(\cdots)$ is zero, hence that $\Omega_{T}(X, \cdots, Z)$ is zero.

We now show that every pair $e_{\alpha}, e_{-\alpha}$ lies in $\mathfrak{t}^{\prime}$ or $\mathfrak{m}^{\prime}$. First, $[H(\mathfrak{m}), \mathfrak{l}]=0$ : For if this were not zero, there would be an element $H \in H(\mathfrak{m})$ and an element $\sum a_{\alpha} e_{\alpha}\left(a_{\alpha}\right.$ complex numbers) in $\mathfrak{l}$ with $\left[H, \sum a_{\alpha} e_{\alpha}\right] \neq 0$; then, since $[\mathfrak{m}, \mathfrak{l}] \subseteq \mathfrak{m}$ and since the semi-simple part of $\mathfrak{l}$ is the semi-simple part of the centralizer of a torus $T$ of $\mathfrak{g}$ (it can easily be seen that our choice of $H\left(\mathfrak{g}^{\prime}\right)$ can be subjected to the condition $\left.T \subseteq H\left(\mathfrak{g}^{\prime}\right) \cap \mathfrak{g}\right)$, it follows that there is an $H^{\prime} \in T$ with $\left[H^{\prime},\left[H, \sum a_{\alpha} e_{\alpha}\right]\right] \neq 0$-i.e. $\sum a_{\alpha} \alpha\left(H^{\prime}\right) \alpha(H) e_{\alpha} \neq 0$, which cannot be since it implies that $\left[H^{\prime}, \sum a_{\alpha} e_{\alpha}\right] \neq 0$. But the condition $[H(\mathfrak{m}), \mathfrak{f}]=0$ implies that $\left[H\left(\mathfrak{m}^{\prime}\right), \mathfrak{f}^{\prime}\right]=0$, hence that the root vectors of the semi-simple part of $\mathfrak{f}^{\prime}$ with respect to $H\left(\mathfrak{f}^{\prime}\right)$ are in fact root vectors of $\mathfrak{g}^{\prime}$ with respect to $H\left(\mathfrak{g}^{\prime}\right)$. Hence it is clear that $\mathfrak{f}^{\prime}$ is spanned by the pairs of root vectors $e_{\alpha}, e_{-\alpha}$ of $\mathfrak{g}^{\prime}$ which lie in $\mathfrak{f}^{\prime}$; and it follows from this that the root vectors of $\mathfrak{g}^{\prime}$ which do not lie in $\mathfrak{l}^{\prime}$, must lie in $\mathfrak{m}^{\prime}$.

Finally, suppose that $G / K$ is a symmetric $C$-space and that rank $G \neq \operatorname{rank} K$. 
Then there exists a nonzero element $H$ in $H\left(\mathfrak{m}^{\prime}\right)$. Let $\alpha$ be a root such that $\alpha(H) \neq 0$. Since $G / K$ is a $C$-space, $e_{\alpha}$ lies in $\mathfrak{t}^{\prime}$ or in $\mathrm{m}^{\prime}$. But the fact that $\alpha(H) \neq 0$ then leads to a contradiction: For example, if $e_{\alpha} \in \mathrm{m}^{\prime}$, then $\left[H, e_{\alpha}\right]$ $=\alpha(H) e_{\alpha}$ is a nonzero element of $\mathfrak{f}^{\prime}\left(\right.$ since $\left.\left[\mathfrak{m}^{\prime}, \mathfrak{m}^{\prime}\right] \subseteq \mathfrak{l}^{\prime}\right)$, which cannot be since $\mathfrak{t}^{\prime} \cap \mathfrak{m}^{\prime}=0$.

13. Maximal abelian subalgebras. Let $K$ be a compact connected Lie group with Lie algebra $\mathfrak{t}$. Let $H(\mathfrak{t})$ be a maximal abelian subalgebra of $\mathfrak{t}$, and let $\mathfrak{l}^{\prime}, H\left(\mathfrak{l}^{\prime}\right)$ be the complexifications of $\mathfrak{t}, H(\mathfrak{l})$. Let $X_{\alpha}, Y_{\alpha}, X_{\beta}, Y_{\beta}, \cdots$ denote the quasi-root vectors of the semi-simple part of $\mathfrak{t}$ with respect to $H\left(\mathfrak{l}^{\prime}\right)$. Let $T$ be an invariant symmetric tensor on $\ell$.

Lemma. Consider any term $T\left(H_{1}, \cdots, H_{j}, X_{\alpha_{1}}, \cdots, X_{\alpha_{n}}, Y_{\beta_{1}}, \cdots, Y_{\beta_{m}}\right)$, with the $H_{i}$ 's elements of $H(\mathfrak{l})$ and the $X_{\alpha_{r}}, Y_{\beta_{r}}$ 's quasi-root vectors of the semisimple part of $\mathfrak{l}$ with respect to $H\left(\mathfrak{f}^{\prime}\right)$. Suppose $\alpha_{1}\left(H_{1}\right) \neq 0$. Then this term can be written as a linear combinations of the following types:

(1) terms $T(\cdots)$ with $j+1$ entries from $H(\mathfrak{t})$;

(2) terms $T(\cdots)$ with $j$ entries from $H(\mathfrak{f})$, with $H_{1}$ in at least two entries.

Proof. The invariance of $T\left(H_{1}, \cdots, H_{j}, H_{1}, X_{\alpha_{2}}, \cdots, Y_{\beta_{1}}, \cdots\right)$ under $Y_{\alpha_{1}}$ shows

$$
\begin{aligned}
0= & i \alpha_{1}\left(H_{1}\right) \cdot T\left(X_{\alpha_{1}}, H_{2}, \cdots, H_{j}, H_{1}, H_{\alpha_{2}}, \cdots, Y_{\beta_{1}}, \cdots\right) \\
& +i \alpha_{1}\left(H_{2}\right) \cdot T\left(H_{1}, X_{\alpha_{1}}, H_{3}, \cdots, H_{j}, H_{1}, X_{\alpha_{2}}, \cdots, Y_{\beta_{1}}, \cdots\right) \\
& \vdots \\
& \cdot \\
& +i \alpha_{1}\left(H_{j}\right) \cdot T\left(H_{1}, \cdots, H_{j-1}, X_{\alpha_{1}}, H_{1}, X_{\alpha_{2}}, \cdots, Y_{\beta_{1}}, \cdots\right) \\
& +i \alpha_{1}\left(H_{1}\right) \cdot T\left(H_{1}, \cdots, H_{j}, X_{\alpha_{1}}, X_{\alpha_{2}}, \cdots, Y_{\beta_{1}}, \cdots\right) \\
& + \text { terms of the form } T\left(H_{1}, \cdots, H_{j}, H_{1}, \cdots\right) .
\end{aligned}
$$

The terms (a) and (b) both equal $\alpha_{1}\left(H_{1}\right) \cdot T\left(H_{1}, \cdots, H_{j}, X_{\alpha_{1}}, \cdots, Y_{\beta_{1}}, \cdots\right)$. Since $\alpha_{1}\left(H_{1}\right) \neq 0$, the lemma is proved by bringing (a) and (b) to the left side of the equality sign.

TheOREM. If $X, \cdots, Z$ are any elements of $\mathfrak{t}$, then $T(X, \cdots, Z)$ can be written as a linear combination (with real coefficients) of terms $T(\cdots)$ all of whose entries are elements of $H(\mathfrak{l})$. Thus the invariant symmetric tensors on $\mathfrak{l}$ depend on the maximal abelian part of $\mathfrak{t}$.

Proof. Consider any term $T\left(H_{1}, \cdots, H_{j}, X_{\alpha_{1}}, \cdots, Y_{\beta_{1}}, \cdots\right)$. It will be shown that this term has property $\mathrm{P}: \mathrm{It}$ is expressible as a linear combination of terms $T(\cdots)$ each having at least $j+1$ entries from $H(\mathfrak{l})$. Thus the proposition will be proved by induction on $j$. We will denote by $\left(^{*}\right)$ any 
linear combination of terms $T(\cdots)$ each having at least $j+1$ entries from $H(\mathfrak{f})$.

Suppose $\alpha_{s}\left(H_{t}\right) \neq 0$ or $\beta_{s}\left(H_{t}\right) \neq 0$ for some $s$ and $t$ (if this is not the case, see Case 2, below); and for simplicity of notation, suppose it is $\alpha_{1}\left(H_{1}\right)$ which is not zero. The preceding lemma shows that $T\left(H_{1}, \cdots, H_{j}, X_{\alpha_{1}}, \cdots, Y_{\beta_{1}}, \cdots\right)$ is expressible (modulo $\left(^{*}\right)$ ) as a linear combination of terms with $H_{1}$ in at least two entries. So we must show that each of the terms in this linear combination has property $\mathrm{P}$. We continue the process described in the lemma until we arrive at a point where the terms are of the form $T\left(H_{1}, \cdots, H_{1}, H_{2}^{\prime}, \cdots\right.$, $\left.H_{k}^{\prime}, X_{\lambda_{1}}, \cdots, Y_{\delta_{1}}, \cdots\right)$, with $\lambda_{1}\left(H_{1}\right)=\lambda_{2}\left(H_{1}\right)=\cdots=\delta_{1}\left(H_{1}\right)=\delta_{2}\left(H_{1}\right)=\cdots$ $=0$. We then apply the process of the lemma to $H_{2}^{\prime}$, and so on, finally arriving at the situation $T\left(H_{1}, \cdots, H_{j}, X_{\alpha_{1}}, \cdots, Y_{\beta_{1}}, \cdots\right)=\left({ }^{*}\right)+\left({ }^{* *}\right)$, with $\left(^{* *}\right)$ a linear combination of terms of the form

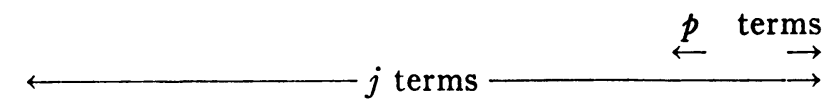

$t=T\left(H_{1}, \cdots, H_{1}, h_{2}, \cdots, h_{2}, \cdots, h_{d}, \cdots, h_{d}, h, \cdots, h, X_{\pi_{1}}, \cdots, Y_{\Delta_{1}}, \cdots\right)$, $h_{2}, \cdots, h_{d}$ elements of $H(\mathfrak{t}), h \in H(\mathfrak{f})$, and $\pi_{r}\left(h_{2}\right)=\cdots=\pi_{r}\left(h_{d}\right)=\Delta_{r}\left(h_{1}\right)=\cdots$ $=\Delta_{r}\left(h_{d}\right)=0$ for all subscripts $r$. So the theorem will be proved if it can be shown that any term $t$ of $\left({ }^{* *}\right)$ has property $\mathrm{P}$.

CASE 1. Suppose that for some $r, \pi_{r}(h) \neq 0$ or $\Delta_{r}(h) \neq 0$; and, for simplicity, suppose it is $\pi_{1}(h)$ which is not zero. Then the invariance of

$$
\stackrel{T\left(H_{1}, \cdots, H_{1}, h_{2}, \cdots, h_{2}, \cdots, h_{d}, \cdots, \underset{h_{d}, h}{\leftarrow}, \cdots, \vec{h}, X_{\pi_{2}}, \cdots, Y_{\Delta_{1}}, \cdots\right)}{\longrightarrow}
$$

under $Y_{\pi_{1}}$ shows that $t$ has property $\mathrm{P}$.

CASE 2. Suppose $\pi_{r}(h)=\Delta_{r}(h)=0$ for all $r$. Choose an $H$ with $\pi_{1}(H) \neq 0$, $H \in H(\mathfrak{t})$. Then the invariance of the following term under $Y_{\pi_{1}}$ shows that $t$ has property $\mathrm{P}$ :

$$
\begin{array}{r}
T\left(H_{1}, \cdots, H_{1}, h_{2}, \cdots, h_{2}, \cdots, h_{d}, \cdots, h_{d}, \stackrel{\left.\stackrel{p}{h}, \cdots, \vec{h}, H, X_{\pi_{2}}, \cdots, Y_{\Delta_{1}}, \cdots\right) .}{\longrightarrow}\right. \\
\stackrel{\text { terms }}{\longrightarrow}
\end{array}
$$

Corollary 1. If rank $G \neq$ rank $K$, the Euler-Poincaré characteristic of $G / K$ is zero. (This is part of the theorem proved in [5].)

Proof. The Gauss-Bonnet theorem shows that it suffices to prove $\Omega_{S}$ is identically zero. This is true by definition if $G / K$ has odd dimension. If the dimension of $G / K$ is $2 N$, choose $H(\mathfrak{l})$ to be a maximal abelian subalgebra of $\mathfrak{l}$ and let $H_{1}, \cdots, H_{N}$ be any elements of $H(\mathfrak{l})$; then $S\left(H_{1}, \cdots, H_{N}\right)$ $=\sum \epsilon\left(\operatorname{ad} H_{1}[\mathrm{~m}]\right)_{i_{1} i_{2}} \cdots\left(\operatorname{ad} H_{N}[\mathrm{~m}]\right)_{i_{2 N-1} i_{2 N}}$. It will be shown that 
$S\left(H_{1}, \cdots, H_{N}\right)$ is zero. It then follows from the preceding theorem that $S$ is identically zero on $\mathfrak{l}$, hence that $\Omega_{S}$ is identically zero. Let $H\left(\mathfrak{t}^{\prime}\right)=H(\mathfrak{l})+i H(\mathfrak{l})$. Extend $H\left(\mathfrak{f}^{\prime}\right)$ to a maximal abelian subalgebra $H\left(\mathfrak{g}^{\prime}\right)$ of $\mathfrak{g}^{\prime}$, and let $H(\mathfrak{m})$ $=H\left(\mathfrak{g}^{\prime}\right) \cap \mathfrak{m}$ and $H\left(\mathfrak{m}^{\prime}\right)=H\left(\mathfrak{g}^{\prime}\right) \cap \mathfrak{m}^{\prime}$. Then $H\left(\mathfrak{g}^{\prime}\right)=H\left(\mathfrak{l}^{\prime}\right)+H\left(\mathfrak{m}^{\prime}\right)$, and $H\left(\mathfrak{m}^{\prime}\right) \neq 0$ since $\operatorname{rank} G \neq \operatorname{rank} K$. If we choose an orthonormal properlyoriented basis $H^{1}, \cdots, H^{s}, X, \cdots, Z$ of $\mathfrak{m}$ (with $H^{1}, \cdots, H^{s}$ in $H(\mathfrak{m})$ ), then, with respect to this basis,

$$
\text { ad } H_{j}[\mathrm{~m}]=\left(\begin{array}{c|c}
0 & 0 \\
\hline 0 & \frac{A_{j}}{}
\end{array}\right) \quad j=1, \cdots, N
$$

with $A_{j}$ a skewsymmetric $(2 N-s) \times(2 N-s)$ matrix.

In the definition of $S\left(H_{1}, \cdots, H_{N}\right)$, one of $i_{1}, \cdots, i_{2 N}$ must in each summand be equal to the number 1 . From the above matrix it can be seen that the corresponding term $\left(\operatorname{ad} H_{j}[\mathrm{~m}]\right)_{1, i_{k}}$ is zero. Thus $S\left(H_{1}, \cdots, H_{N}\right)=0$.

CoRollaRy 2. If rank $G=$ rank $K$, the natural mapping of $I(\mathfrak{g})$ into $I(\mathfrak{l})$ is $1-1$.

Proof. Choose any maximal abelian subalgebra of $g$ which lies entirely in $\mathfrak{t}$. The preceding theorem shows that any element of $I(\mathfrak{g})$ is then determined by its values on $\mathfrak{t}$.

\section{BiBLIOGRAPHY}

1. W. Ambrose and I. M. Singer, A theorem on holonomy, Trans. Amer. Math. Soc. vol. 75 (1953) pp. 428-443.

2. H. Cartan, Colloque de topologie (espaces fibres), Bruxelles, 1950, Liège et Paris, 1951, pp. 15-27 and pp. 57-71.

3. S. S. Chern, Topics in differential geometry, Princeton, Institute for Advanced Study, 1951.

4. E. Dynkin, The structure of semi-simple Lie algebras, Amer. Math. Soc. Translations, no. $17,1950$.

5. H. Hopf and H. Samelson, Ein Satz über die Wirkungsraume geschlossener Leischer Gruppen, Comment. Math. Helv. vol. 13 (1940-1941) pp. 240-251.

6. K. Nomizu, Invariant affine connections on homogeneous spaces, Amer. J. Math. vol. 76 (1954) pp. 33-65.

7. H. Samelson, Topology of Lie groups, Bull. Amer. Math. Soc. vol. 58 (1952) pp. 2-37.

8. H. C. Wang, Closed manifolds with homogeneous complex structure, Amer. J. Math. vol. 76 (1954) pp. 1-32.

University of California,

Los Angeles, Calif. 